médecine/sciences $1997 ; 13: 749-52$

\title{
COURRIER
}

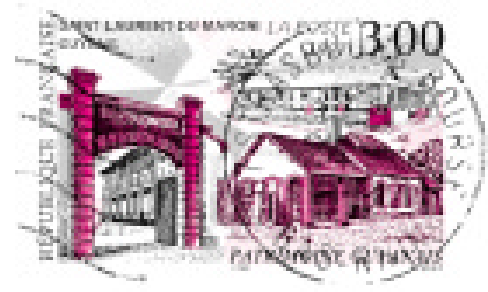

\section{Exposition à de faibles niveaux d'amiante et risques de cancer: plausibilité scientifique et problèmes de santé publique}

Dans une lettre publiée dans le numéro de mars de médecine/sciences [1], le Pr Étienne Fournier exprime un ensemble de points de vue inspirés par le rapport de l'Expertise scientifique collective de l'Inserm consacrée aux effets sur la santé des expositions à l'amiante [2]. Le PrFournier aborde des problèmes, scientifiques et de santé publique, qui concernent divers aspects des effets de l'exposition à l'amiante. Nous souhaitons, à notre tour, apporter notre point de vue sur certains de ces problèmes. Quelques-uns concernent des aspects relativement ponctuels, mais pour lesquels il convient néanmoins d'apporter quelques éclaircissements, car ils peuvent avoir d'importantes conséquences de santé publique. Ainsi, concernant le rôle de l'amiante vis-à-vis du cancer du poumon, toutes les études montrent que l'amiante a un effet cancérogène propre pour le poumon, chez les fumeurs comme chez les non-fumeurs $[2,3]$. Le Pr Fournier considère également que la nocivité des amphiboles «est reconnue être bien supérieure à celle du chrysotile». Si cette affirmation est bien établie pour le risque de mésothéliome, rien ne permet de l'affirmer pour ce qui concerne le cancer du poumon; il est au contraire, clairement établi que l'effet cancérogène de fibres d'amiante d'appellation commerciale "chrysotile» est du même ordre de grandeur que celui des fibres de type amphibole pour le poumon [4]. Or, dans une population exposée à l'amiante, le nombre de cas de cancer du poumon attribuables à ce facteur est plus élevé que le nombre de mésothéliomes: laisser croire que le chrysotile serait moins dangereux pourrait donc avoir de graves conséquences en termes de santé publique.

Le problème du seuil: impossibilité d'observer ou impossibilité d'exister?

Le point le plus important évoqué par le Pr Fournier concerne l'existence d'une dose-seuil qui, selon lui, est un principe qui n'admet aucune exception lorsqu'il s'agit d'effets toxiques, "quel que soit le toxique, quel que soit l'effet à évaluer». Le raisonnement du Pr Fournier semble reposer sur le fait qu'en deçà de certaines doses de produits toxiques, il n'est pas possible d'observer un effet. Si on comprend bien le raisonnement du Pr Fournier, ne pas observer d'effet en deçà d'une certaine dose obligerait à admettre que cette dose est située en deçà d'un «seuil », c'està-dire qu'aucun effet n'existerait, en aucune circonstances, en dessous de cette dose. Le Pr Fournier propose donc d'assimiler absence d'observation dans une ou des études avec inexistence dans l'absolu. En fait, le point soulevé par le Pr Fournier doit se décomposer en deux questions distinctes qui se suivent logiquement: (1) l'exposition à un facteur (disons l'amiante) est-elle susceptible de provoquer un effet toxique (existe-t-il une relation de cause à effet) ?; (2) si oui, cette relation existe-t-elle aussi bien aux doses élevées qu'aux faibles doses ou comporte-t-elle un seuil?

Méthodes de mise en évidence de l'existence d'une relation de causalité

Si pour certains risques (chute d'une hauteur élevée, intoxication mortelle par ingestion de certains champignons) on peut établir sans ambiguïté une relation de cause à effet individuelle entre le facteur de risque et l'effet, il n'en est pas de même pour d'autres risques. Ainsi, pour le cancer du poumon associé au tabac, pour les leucémies associées 
aux rayonnements ionisants ou au benzène, etc., on ne dispose d'aucun élément permettant d'établir une relation de causalité au niveau individuel: alors même que la possibilité de déclencher l'effet par l'exposition à ces facteurs est avérée, rien ne permet de distinguer un des cancers cités qui leur serait dû, d'une tumeur dont l'apparition serait liée à d'autres causes, connues ou inconnues.

Les méthodes expérimentales, in vitro et in vivo, visent à provoquer l'effet toxique soupçonné en administrant le facteur étudié de façon contrôlée: si l'effet apparaît, on peut alors l'attribuer de façon fiable au facteur. Mais chacun sait que le passage du modèle cellulaire ou animal à l'homme est tout à fait incertain, et une confirmation épidémiologique est nécessaire pour attribuer une action causale à un facteur vis-à-vis d'une maladie.

La démarche scientifique adoptée par l'épidémiologie vise précisément à montrer que les populations humaines exposées au facteur présentent ou pas un niveau de risque plus élevé que les populations non exposées et à réunir les observations permettant d'étayer ou d'infirmer l'hypothèse selon laquelle l'exposition des populations étudiées au facteur de risque est reliée de façon causale à l'augmentation du risque dans ces populations. Rappelons au Pr Fournier que pour juger de la cancérogénicité d'un facteur de risque pour l'homme, le Centre International de Recherche sur le Cancer considère que les résultats épidémiologiques priment sur les résultats expérimentaux [5].

\section{L'amiante est cancérogène aux doses «élevées »}

Concernant l'amiante, des données expérimentales et des études épidémiologiques innombrables ont confirmé le caractère cancérogène de l'exposition à ce facteur [2]. Les données épidémiologiques actuellement disponibles ne montrent des risques significativement accrus qu'à partir de niveaux d'exposition relativement élevés, mais néanmoins sensiblement plus faibles que ne l'indique le Pr Fournier. Des études récentes concernant des expositions professionnelles montrent un excès statistiquement significatif de mésothéliome à partir d'une exposition cumulée de 5 (fibres $/ \mathrm{ml}$ ) $x$ année [6], ou un excès statistiquement significatif de cancer du poumon chez des travailleurs exposés de façon intermittente [7], ou à des niveaux cumulés de 8 (fibres/ml) $x$ année [8]. Ces études montrent clairement que c'est parmi les catégories de personnel exposées de façon sporadique à l'occasion de l'exécution de travaux d'intervention courants sur des matériaux contenant de l'amiante qu'on observe aujourd'hui le plus grand nombre de mésothéliomes [9]. On dispose également aujourd'hui d'études concernant des populations résidant au voisinage de mines d'amiante et ayant été exposées uniquement de façon «environnementale » au Québec [10] et en Australie [11]: ces études montrent un risque nettement et significativement accru de mésothéliome (notamment dans des populations féminines n'ayant jamais occupé d'emploi dans l'industrie de l'amiante), correspondant à des niveaux d'exposition relativement peu élevés.

\section{L'impossibilité intrinsèque de mettre en évidence de façon "certaine" des effets faibles associés à des doses faibles}

Mais si l'affinement des études épidémiologiques récentes permet de mettre en évidence des excès de risque statistiquement significatifs correspondant à des expositions sensiblement plus faibles que pour les études plus anciennes, il faut cependant insister, comme nous l'avons fait dans le rapport Inserm, sur l'impossibilité intrinsèque de mettre en évidence l'effet de faibles doses d'amiante tant par des études expérimentales chez l'animal que par des études épidémiologiques dans des populations humaines.

Ceci est notamment dû à des problèmes de «puissance statistique", qui semblent inconnus du Pr Fournier. Il s'agit pourtant d'un élément essentiel de la démarche scientifique. Tout montre [2] que pour l'amiante il existe aux niveaux d'exposition "élevés» (> 1 fibre $/ \mathrm{ml}$ ) une relation croissante et bien décrite sur le plan quantitatif entre le niveau d'exposition et le risque de cancer: celui-ci est d'autant plus élevé que le niveau d'exposition est élevé et s'avère dépendre linéairement de l'exposition cumulée à l'amiante $(\mathrm{f} / \mathrm{ml} \mathrm{x}$ année). Il est donc logique de s'attendre à ce qu'en deçà de ces niveaux, le risque, s'il existe, soit plus faible.

Or, il est intuitif que plus le pouvoir cancérogène du facteur étudié est élevé, et plus grande sera la probabilité d'observer un risque significativement accru parmi les sujets exposés, et inversement. Un deuxième paramètre joue un rôle important: à risque égal, plus le nombre de sujets (personnes ou animaux) inclus dans une étude est élevé, plus grande est la probabilité de détecter un accroissement de risque statistiquement significatif.

En pratique un expérimentateur devrait disposer, pour détecter d'éventuels faibles risques associés à des faibles doses, d'un nombre d'animaux qu'il serait illusoire de vouloir réunir. Il en va de même dans un contexte épidémiologique. Pour illustrer ce fait nous avons calculé, dans le cadre de l'expertise de l'Inserm, la probabilité d'observer un excès de risque statistiquement significatif dans différents types d'études lorsque l'excès de risque est égal à 1 cas supplémentaire de cancer du poumon pour 1000 personnes exposées dans la population représentée par cette étude. Un tel risque, "faible » selon des critères épidémiologiques, n'est pas négligeable d'un point de vue de santé publique, et pourrait correspondre d'après le modèle d'extrapolation des fortes aux faibles doses, si vertement critiqué par le PrFournier, au risque associé à une exposition approximativement égale à 100 fibres/litre (valeur de référence actuelle pour la limitation réglementaire des expositions professionnelles). Le calcul montre que dans des conditions d'enquête «parfaites» (totalement impossibles à assurer dans la pratique) le suivi d'une cohorte de 10000 sujets depuis l'âge de 20 ans jusqu'à celui de 80 ans aurait moins 
de $15 \%$ de chances de mettre en évidence un accroissement de risque statistiquement significatif. Si on voulait atteindre une probabilité considérée comme satisfaisante selon les critères scientifiques habituels $(95 \%$ de chances de détecter un tel accroissement) c'est une cohorte de plus de 325000 sujets présentant exactement ces conditions d'exposition qu'il faudrait réunir et suivre pendant 60 ans ! On comprend que de telles études sont impossibles à réaliser et que même s'ils existent, des effets faibles n'ont donc qu'une très faible probabilité de donner lieu à des observations statistiquement significatives dans le cadre d'études réalisables sur le plan pratique. Affirmer alors, en prétendant être dépositaire de la certitude scientifique, comme le fait le Pr Fournier, qu'à coup sûr de tels effets ne peuvent exister puisqu'on ne les a pas observés, est une conclusion dont nous lui laissons la responsabilité scientifique et éthique. Soulignons à ce propos que l'hypothèse de l'existence d'un seuil d'innocuité de l'amiante et celle d'une relation dose-risque de forme identique aux fortes et aux faibles doses ont un rôle parfaitement symétrique et sont, en somme, indiscernables: s'il est en effet impossible de démontrer de façon certaine que le modèle d'extrapolation des fortes aux faibles doses est plus valide que celui de l'existence d'un seuil, il est tout aussi impossible, et pour les mêmes raisons, de démontrer que l'hypothèse de l'existence d'un seuil d'innocuité est plus valide que celle d'une unique forme de relation dose-risque des fortes aux faibles doses.

\section{L'absence de seuil : hypothèse, certes « incertaine", mais la plus «plausible»}

Nous sommes ici au cœur du problème essentiel posé par la gestion $\mathrm{du}$ risque amiante, puisque de larges fractions de la population sont exposées à des niveaux «faibles»: devant l'impossibilité de démontrer, de façon certaine, l'existence ou l'inexistence d'un risque pour ces populations, quelle est la meilleure attitude à adopter? Tout d'abord, il faut, sur la base d'arguments scienti- fiques, envisager la vraisemblance de l'existence d'un risque à des doses dites «faibles", c'est-à-dire d'un risque à propos duquel les connaissances ne peuvent qu'être incertaines, comme on vient de le voir. Dans le cas de l'amiante, l'opinion du groupe de scientifiques réuni par l'Inserm, établie en tenant compte de plusieurs décennies de résultats scientifiques de toutes natures (on ne peut à l'évidence considérer l'amiante comme d'autres facteurs pour lesquels on n'a pas d'indication positive de leur cancérogénicité, ou même pour lesquels on ne dispose pas d'une telle accumulation de données scientifiques), a été de considérer comme les hypothèses les plus plausibles l'existence d'un tel risque et la continuité de la forme de la relation dose-risque (linéarité du risque par rapport à l'exposition cumulée) des fortes aux faibles doses.

Épidémiologie et disciplines biologiques se rejoignent en effet pour considérer aujourd'hui que le modèle incertain le plus plausible pour la cancérogenèse est celui d'une relation sans seuil. La plupart des tumeurs malignes prennent leur origine dans la transformation d'une unique cellule, et une dose très faible d'un cancérogène est tout à fait capable d'induire une mutation transformante dans le patrimoine génétique d'une cellule. Chaque cellule a donc une probabilité extrêmement faible de donner lieu au développement d'une tumeur (nos organes sont composés de millions de cellules et une infime fraction d'entre elles sont transformées par l'exposition à un cancérogène), mais cette probabilité n'est pas nulle. En l'absence de tout modèle alternatif plus convaincant, c'est le modèle de la proportionnalité simple entre la dose et le risque de mutation transformante d'une unique cellule qui est, contrairement aux affirmations $\mathrm{du} \operatorname{Pr}$ Fournier, très largement adopté de nos jours. Dans le cas de l'exposition à l'amiante, ce modèle s'ajuste de façon tout à fait satisfaisante aux observations épidémiologiques directes faites aux "fortes» expositions $(>1 \mathrm{f} / \mathrm{ml})$, son extrapolation aux faibles doses $(<1 \mathrm{f} / \mathrm{ml})$ concerne un domaine d'incertitude mais est très généralement considérée comme la plus plausible sur le plan scientifique.

\section{Les conséquences d'un risque vraisemblablement associé à des faibles doses}

Si on considère vraisemblable l'existence d'un risque à des doses «faibles », il est légitime, en l'absence de données d'observation directes, de tenter d'estimer l'impact «théorique» (terme que nous préférons à celui d'imaginaire utilisé par le Pr Fournier) de telles expositions dans la population, afin d'apporter des éléments de cadrage, certes incertains, mais qui nous sont apparus les plus plausibles, dans l'état actuel des connaissances. C'est ce que nous avons fait, à la suite d'autres groupes d'experts étrangers (qui comprenaient d'ailleurs des toxicologues), en ne manquant pas de souligner, répétitivement, qu'il s'agissait d'estimations, fondées sur des hypothèses, les plus plausibles dans l'état actuel des connaissances, mais, néanmoins, incertaines. De tels exercices de modélisation sous incertitude sont courants dans de nombreux domaines de la santé ou de l'économie, par exemple, afin d'éclairer les décideurs sur les conséquences possibles de leurs choix.

Il faut bien sûr aussi, lorsqu'on considère vraisemblable l'existence d'un risque à des doses "faibles", envisager les mesures à prendre. En n'oubliant pas, faut-il le rappeler, que plus la dose est faible, plus le risque est faible: il est certainement tout à fait discutable de considérer $a$ priori que l'existence d'un risque "sans seuil» doive obligatoirement entraîner l'éradication totale du facteur de risque dans le but de parvenir au «risque zéro", ce qu'aucun spécialiste de santé publique ne peut considérer sérieusement. Ainsi, de nombreux facteurs considérés comme des cancérogènes avérés font l'objet d'une réglementation visant, non pas à les supprimer totalement, mais à fixer des valeurs limites d'exposition pour les travailleurs ou le public. Il est intéressant de noter ici que les méthodes formalisées de détermination de valeurs d'exposition «tolé- 
rables » utilisent fréquemment les concepts de "no observed effect limit» (NOEL) et de "facteur de sécurité»: pour proposer une valeur limite aux expositions humaines considérée comme "tolérable», on divise le NOEL observé en expérimentation par divers facteurs de sécurité afin de minimiser la probabilité que des effets se produisent chez l'homme [12]. Il est par exemple courant de diviser une première fois le NOEL par 10 pour tenir compte du passage de l'animal à l'homme, une seconde fois par 10 pour tenir compte de la variabilité interindividus et une dernière fois par 10 pour adopter une marge de sécurité. Cette démarche souligne bien que le fait de ne pas observer d'effet n'est nullement considéré comme la preuve de l'inexistence d'un effet. Il est également intéressant de souligner que cette démarche, courante en toxicologie, si elle était appliquée aux expositions à l'amiante, risquerait fort d'aboutir à des valeurs limites d'exposition plus sévères que les valeurs de références actuelles. Il n'y a d'ailleurs là rien d'étrange car à clamer bien fort l'évidence de l'existence d'un seuil d'innocuité on ne résoud pas le problème, autrement difficile, d'en proposer une estimation scientifiquement crédible! Le «principe de précaution" adopté fort heureusement de longue date par les toxicologues pour la meilleure protection de tous consiste à répondre de façon tout à fait empirique à ce défi (généralement insoluble sur le plan scientifique) en adoptant une très exigeante cascade de «facteurs de sécurité" à partir d'un NOEL. Sur un plan tout à fait pratique, la démarche du seuil et de la cascade de facteurs de protection et celle de l'extrapolation épidémiologique des fortes aux faibles doses finissent ainsi par se ressembler fort en termes de valeurs d'exposition tolérables.

En pratique, les mesures de prévention à prendre peuvent être très variées selon les circonstances, et de très nombreuses considérations d'ordre éthique, sanitaire, social, économique, technologique notamment doivent être prises en compte. C'est là que le débat social, transparent et
Il nous semble que la contribution de l'expert scientifique doit se cantonner à son rôle spécifique, qui est d'établir les éléments scientifiques les plus pertinents et les mieux validés qu'il est possible de le faire à un moment donné de la connaissance, et les mettre à la disposition de chacun de ceux qui doivent participer au débat social. Il est essentiel que celuici ait lieu: c'est pourquoi la prise de position du Pr Fournier nous semble finalement un élément important et susceptible de le faire progresser. A condition, bien sûr, de respecter la rigueur scientifique qui s'impose là comme ailleurs: comme le Pr Fournier, nous pensons que "selon les règles de l'éthique et de la déontologie médicale (et même du droit commun), il revient aux scientifiques de refuser de proposer aux médias des informations contestables»

\section{RÉFÉRENCES}

Bien que cette lettre ait été rédigée par des "personnes disposant déjà de leur bibliographie", tout comme le Pr Fournier, il nous a semblé que les lecteurs pouvaient souhaiter se reporter eux-mêmes aux références citées, comme cela était jusqu'à présent usuel dans le contexte de la communication scientifique.

1. Fournier PE. Application des principes de la toxicologie aux faibles niveaux d'amiantes. Med Sci 1997; 13: 422-3.

2. Inserm. Effets sur la santé des principaux types d'exposition à l'amiante. Expertise Collective. Rapport de synthèse, Inserm, Paris, Juin 1996. (Rapport complet sous presse.)

3. IARC Monographs on the evaluation of carcinogenic risk of chemicals to man. International Agency for Research on Cancer, Lyon, 1977, vol. 14.

4. Stayner LT, Dankovic DA, Lemen RA. Occupational exposure to chrysotile asbestos and cancer risk: a review of the amphibole hypothesis. Am J Pub Health 1996; 86 : 179-86.

5. IARC Monographs on the evaluation of carcinogenic risk of chemicals to humans. Overall evaluations of carcinogenicity: an updating of IARC monographs Vol. 1 to 42. International Agency for Research on Cancer, Lyon, 1987 (suppl 7).
6. Iwatsubo Y, Pairon JC, Boutin C, Martinet Y, Caillaud D, Bignon J, Brochard P. Pleural mesothelioma: dose-response relationship at low level of asbestos exposure in a population-based case-control study. Am J Epidemiol 1997 (sous presse).

7. Ahrens W, Jöckel KH, Pohlabeln H, et al. Assessment of exposure to asbestos in a case-control study of lung cancer: comparison of supplementary questionnaires an an exposure check-list. Occup Hygiene 1996; 3 : 125-36.

8. Imbernon E, Goldberg M, Bonenfant S, et al. Occupational respiratory cancer and exposure to asbestos: a case-control study in a cohort of workers in the electricity and gas industry. Am J Indust Med 1995 ; 28 : 33952 .

9. Peto J, Hodgson JT, Matthews FE, Jones JR. Continuing increase in mesothelioma mortality in Britain. Lancet 1995; 345 : 535-9.

10. Camus M, Siemiatycki J, Dewar R. Nonoccupational asbestos exposure and risk of lung cancer in the female population of asbestos mining towns: implications for risk assessments (communication personnelle).

11. Hansen J, de Klerk NH, Musk AW, Hobbs MST. Environmental exposure to crocidolite and mesothelioma: exposureresponse relationships. Am J Resp Crit Care Med 1997 (sous presse).

12. Bard D. Principes de l'évaluation des risques pour la santé publique liés aux expositions environnementales. Rev Epidemiol Sante Publ 1995; 43: 423-31.

\section{Marcel Goldberg}

Professeur à l'Université Paris $V$, directeur de l'Unité Inserm U. 88, Épidémiologie, Santé publique et environnement professionnel et général, Hôpital national de Saint-Maurice, 14, rue du Val-d'Osne, 94415 Saint-Maurice Cedex, France.

\section{Denis Hémon}

Directeur de recherche à l'Inserm, Directeur de l'Unité Inserm U. 170, Recherches épidémiologiques et statistiques sur l'environnement et la santé, 16, avenue PaulVaillant-Couturier, 94807 Villejuif Cedex, France.

Rapporteurs de l'expertise scientifique collective de l'Inserm: "Effets sur la santé des principaux types d'exposition à l'amiante. » 